\title{
Review Article \\ Review of Clinical Studies of the Treatment of Ulcerative Colitis Using Acupuncture and Moxibustion
}

\author{
Jun Ji, ${ }^{1}$ Yan Huang, ${ }^{1,2}$ Xia-Fei Wang, ${ }^{3}$ Zhe Ma, ${ }^{3}$ Huan-Gan Wu, ${ }^{1,2}$ Hyoyoung Im, ${ }^{3}$ \\ Hui-Rong Liu, ${ }^{1,2}$ Lu-Yi Wu, ${ }^{2}$ and Jing $\mathrm{Li}^{4}$ \\ ${ }^{1}$ Shanghai Research Institute of Acupuncture and Meridian, Shanghai University of Traditional Chinese Medicine, \\ Shanghai 200030, China \\ ${ }^{2}$ Key Laboratory of Acupuncture-Moxibustion and Immunology, Shanghai University of Traditional Chinese Medicine, \\ Shanghai 200030, China \\ ${ }^{3}$ Yueyang Clinical Medical College, Shanghai University of Traditional Chinese Medicine, Shanghai 200437, China \\ ${ }^{4}$ Department of Acupuncture and Moxibustion, Shanghai Yueyang Hospital of Integrated Traditional Chinese and Western Medicine, \\ Shanghai University of Traditional Chinese Medicine, Shanghai 200437, China
}

Correspondence should be addressed to Lu-Yi Wu; luyitcm@163.com and Jing Li; 1971921250@qq.com

Received 22 August 2016; Accepted 10 October 2016

Academic Editor: Paolo Gionchetti

Copyright (C) 2016 Jun Ji et al. This is an open access article distributed under the Creative Commons Attribution License, which permits unrestricted use, distribution, and reproduction in any medium, provided the original work is properly cited.

\begin{abstract}
Background. Clinical studies suggest that acupuncture and moxibustion therapy in ulcerative colitis (UC) can regulate bowel inflammation, and these treatments have the advantages of low rates of adverse reactions and recurrence as well as good long-term efficacy. We reviewed the current status of clinical studies of the treatment. Methods. Randomized controlled trials (RCTs) using the therapy as the major intervention for treating UC were included from 1995 to 2015 . The extracted data mainly included diagnostic standards, treatment methods, selection of acupoints, treatment times and courses, and efficacy determination criteria. Results. The use of diagnostic standards and efficacy criteria lacked unification and standardization. There were two main groups: acupuncture and moxibustion therapy combined with drug treatment and the use of all types of acupuncture and moxibustion therapy alone or in combination. The acupoint compositions included distal-proximal point combinations, back-shu point and front-mu point combinations, and acupuncture through meridians. The treatment courses in all the clinical trials had large variations. Conclusion. The treatment of UC in the examined articles was mainly based on the classical theory. However, many links of the clinical regimen design were still lacking, which affected the repeatability of the clinical studies and the accuracy of the clinical conclusions.
\end{abstract}

\section{Introduction}

Ulcerative colitis (UC) is a chronic, nonspecific inflammatory disease with unknown etiology. UC mainly invades the distal colon, rectal mucosa, and submucosa, and it can involve the entire colon or even the terminal ileum. Its clinical manifestations are mainly recurrent mucopurulent bloody stool, abdominal pain, diarrhea, and combined systemic presentations to different degrees. The disease course is very long which can result in the formation of intestinal fibrosis and stenosis. The age of disease onset is mainly young adults aged 20-40 years. There is no difference between males and females. Generally, the disease has slow development and different severity and is prone to recurrence. Some disease courses can last for several years or even several decades. UC is closely associated with the development of colon cancer and is recognized by the World Health Organization (WHO) as a refractory bowel disease.

The pathogenic mechanism of UC is still not completely clear. Modern medicine considers the development of UC to be associated with a variety of factors, mainly including environmental factors, immune factors, inflammation, eating disorders, emotional distress, and genetics. Currently, there is no radical therapy for UC. UC is mainly treated with aminosalicylic acid preparations \{salazosulfapyridine $(S A S P)$ and aminosalicylic acid (ASA)\}, steroid hormones, immunosuppressants, and new biologics. The goal of treatment is to maximally relieve symptoms, eliminate inflammation, heal 
ulcers, prevent complications, and prevent recurrence. However, the efficacy of treatment using the above drugs has large individual differences, and there are different levels of toxic side effects.

Based on its clinical manifestations, this disease can be classified into the scope of "intestinal afflux," "diarrhea," "chronic diarrhea," "chronic dysentery," and "bloody stool" in traditional Chinese medicine. Symptoms are mainly caused by feeling pathogenic dampness, improper diet, emotional disorders, and weakness of the viscera. Therefore, the disease location is mainly the large intestine and is closely associated with the spleen and stomach; in addition, UC usually involves the liver and kidney. Currently, the treatment of this disease using traditional Chinese medicine mainly involves therapeutic methods such as the administration of traditional Chinese medicines orally or via enemas, acupuncture, and moxibustion. Since the 1990s, clinical studies on the treatment of UC using acupuncture and moxibustion have gradually increased. Current studies show that acupuncture and moxibustion can regulate physiological balance at multiple links and multiple targets in the body to effectively control bowel inflammation. In addition, these treatments have the advantages of low adverse reactions, low recurrence, and good long-term efficacy. Therefore, these treatments have been applied extensively.

A review of clinical studies in this field based on randomized controlled trials (RCTs) of UC treatment using acupuncture and moxibustion published between 1999 and 2015 was performed, and the results are described below.

\section{Materials and Methods}

2.1. Retrieval Strategy. The searched databases included foreign language databases and Chinese databases. Foreign literature on registered RCTs was searched in Medline, EMBASE, and the Cochrane Library. The search was limited to human studies and RCTs. Chinese literature was searched in the following databases: the China National Knowledge Infrastructure Database (CNKI), the Chongqing VIP Chinese Science and Technology Periodical Database (VIP), and the Chinese Biomedical Literature Database (CBM). The search period was from January 1, 1995, to December 31, 2015. The Chinese Medical Subject Headings (MeSH) terms included "inflammatory bowel disease", "ulcerative colitis", "acupuncture", "moxibustion", "acupoint", and "acupuncture and moxibustion therapy". The English MeSH terms were "acupuncture", "moxibustion", "inflammatory bowel disease", and "ulcerative colitis". According to the specific conditions of the different databases, a comprehensive retrieval using MeSH terms combined with key words and free words was performed to ensure the completeness of the search results.

2.2. Inclusion Criteria. (1) Study subjects: patients with a definitive diagnosis of UC were included. There were no restrictions on race, age, or gender. (2) Study design: RCTs of UC treatment using acupuncture and moxibustion were included. The languages were limited to Chinese and English. (3) Intervention measures of experimental groups: experimental groups mainly received acupuncture and moxibustion therapy (including filiform needle acupuncture, electroacupuncture, moxibustion, and cupping therapy) alone or combined with other treatment methods (such as drug therapy). In addition, needling methods, acupoint selection, and needle material were not further classified. For acupuncture and moxibustion combined with drug therapy, the drugs administered to experimental groups and control groups in the same study should be consistent. (4) There were no restrictions on the intervention measures used in control groups. (5) Literature with full articles or abstracts that provided sufficient information was included.

2.3. Exclusion Criteria. Literature was excluded due to the following conditions: (1) study subjects and intervention measures that did not conform to the inclusion criteria; (2) RCTs without clear diagnostic standards, basic information on subjects, or intervention measure-related information; (3) series of observations, case reports, expert experiences, and descriptive analyses without controlled cases; and (4) literature with duplicated detection or duplicated publication.

2.4. Data Extraction. Two researchers independently performed literature screening, modified Jadad quality scoring, data information extraction, and database establishment. A third assessor checked the data for consistency. Cases of inconsistent data were resolved by discussion. The extracted data mainly included the number of published studies, diagnostic standards, treatment methods and results, acupoint and meridian selection, treatment times and courses, and efficacy determination criteria.

\section{Results}

According to the retrieval strategy, a total of 872 Chinese and English literature reports were retrieved from the above medical databases. A bibliography of the literature was introduced into Excel, and repeated bibliographies were deleted. After reading titles and abstracts, significantly unrelated articles were excluded. The full articles were downloaded and read. Unrelated articles were deleted according to the study's literature exclusion criteria. Finally, 63 articles were included [163]. These articles included 58 Chinese articles and 5 English articles. The literature types included journal articles and graduate student degree dissertations. The number of clinical study articles on this disease was stable every year. The lowest annual number was 0 (2002), and the highest number was 10 (2015). Overall, the numbers showed a rising trend (Figure 1).

3.1. Diagnostic Standards. Summary statistics was performed on the 63 included UC articles. The results showed that 58 of the articles described the diagnostic standards that were used, whereas 5 articles [25, 29, 30, 47] did not explain these standards. Among the diagnostic standards adopted by these 58 articles, consensus opinions formulated by Chinese industry associations or expert committees were used in 8 articles, the practical guidelines for the diagnosis and treatment of inflammatory bowel diseases of the World Gastroenterology Organization were used in 1 article, and the other articles used standards from different types of teaching materials and 
TABLE 1: The use of diagnostic standards.

\begin{tabular}{|c|c|c|}
\hline $\begin{array}{l}\text { Serial } \\
\text { number }\end{array}$ & Diagnostic standards & $\begin{array}{c}\text { Number of } \\
\text { articles }\end{array}$ \\
\hline 1 & $\begin{array}{l}1978 \text { Hangzhou conference on ulcerative diagnostic standards of the Chinese Society of Gastroenterology, } \\
\text { Chinese Medical Association }\end{array}$ & 4 \\
\hline 2 & $\begin{array}{l}1993 \text { Taiyuan ulcerative colitis diagnostic criteria from the national conference on chronic noninfectious } \\
\text { intestinal diseases }\end{array}$ & 10 \\
\hline 3 & $\begin{array}{l}2000 \text { Chengdu conference on diagnostic standards for inflammatory bowel disease of the Chinese Society of } \\
\text { Gastroenterology, Chinese Medical Association }\end{array}$ & 13 \\
\hline 4 & $\begin{array}{l}2003 \text { Chongqing integrative diagnostic and treatment programs for ulcerative colitis of the professional } \\
\text { committee on digestive system diseases of the Chinese Association of Integrative Medicine }\end{array}$ & 2 \\
\hline 5 & $\begin{array}{l}2007 \text { consensus on standardization of diagnosis and treatment of inflammatory bowel disease in China by the } \\
\text { inflammatory bowel disease collaborative group of the Chinese Society of Gastroenterology, Chinese Medical } \\
\text { Association }\end{array}$ & 3 \\
\hline 6 & $\begin{array}{l}2009 \text { consensus on diagnosis and treatment of ulcerative colitis using traditional Chinese medicine of the } \\
\text { Digestive Diseases Branch of China Association of Traditional Chinese Medicine }\end{array}$ & 2 \\
\hline 7 & $\begin{array}{l}2010 \text { consensus on diagnosis and treatment of ulcerative colitis using traditional Chinese medicine of the } \\
\text { Digestive Diseases Branch of China Association of Traditional Chinese Medicine }\end{array}$ & 2 \\
\hline 8 & $\begin{array}{l}2010 \text { practical guidelines for the diagnosis and treatment of inflammatory bowel disease of the World } \\
\text { Gastroenterology Organization }\end{array}$ & 1 \\
\hline 9 & $\begin{array}{l}2010 \text { consensus on integrative diagnosis and treatment of ulcerative colitis of the professional committee on } \\
\text { digestive system diseases of the Chinese Association of Integrative Medicine }\end{array}$ & 2 \\
\hline 10 & Teaching materials, different types of books, and other sources & 18 \\
\hline 11 & Self-formulated standards & 10 \\
\hline 12 & No diagnostic standards & 5 \\
\hline
\end{tabular}

Note: A total of 9 articles adopted both traditional Chinese medicine and Western medicine standards. "Teaching materials, different types of books, and other sources" included 3 articles on "internal medicine," 1 article on the industry standards of the State Administration of Traditional Chinese Medicine, 3 articles on "principles of guidelines for clinical studies of new drugs in traditional Chinese medicine," 1 article on "basics of Western internal medicine," 1 article on "diagnostic and treatment standards of symptoms and prescription selection in traditional Chinese medicine," 3 articles on "practical internal medicine," 1 article on "clinical diagnostic and treatment guidelines" of the Chinese Medical Association, 1 article on "diagnostics in traditional Chinese medicine," 1 article on "complete modern acupuncture therapy," 1 article on "basics of Western internal medicine," 1 article on "diagnostic analysis and diagnosis of digestive diseases," and 1 article of English literature.

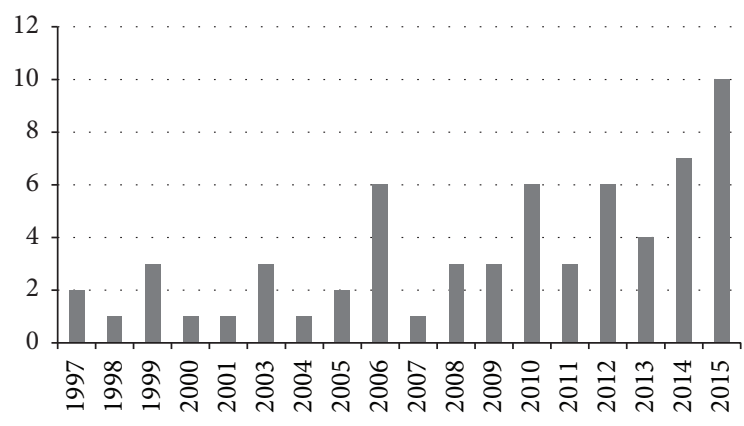

FIGURE 1: Number of clinical study articles.

books. The two most frequently used diagnostic standards were the "1993 ulcerative colitis diagnostic criteria from the Taiyuan National conference on chronic noninfectious intestinal diseases" and the "2000 diagnostic standards from the Chengdu conference on inflammatory bowel disease of the Chinese Society of Gastroenterology, Chinese Medical Association." For the past 2-3 years, the definitive diagnosis of UC has been divided into Western medicine diagnosis and traditional Chinese medicine diagnosis; the latter focuses on the confirmation of the classification of symptom differentiation. In the past, only one type of diagnostic standard was used; there were diverse types of standards, which were not restricted to conference consensuses. Moreover, domestically formulated standards were generally used; only 1 article used foreign standards [61] (Table 1).

3.2. Use of Acupuncture and Moxibustion Therapy. A statistical analysis of the intervention measures used in the experimental groups of the 63 included articles showed these measures were efficacy and mainly of two types. One type was acupuncture and moxibustion therapy combined with drug treatment. The drugs could be either Western medicines or traditional Chinese medicines. A small number of articles used combined Western and traditional Chinese medicines. The drug administration methods included oral, enema, and rectal administration. The other type of intervention measure was the use of acupuncture and moxibustion alone or in combination. The most commonly used acupuncture and moxibustion therapies included acupuncture, electroacupuncture, moxibustion, warm needling, acupoint catgut embedding, acupoint application, balance cupping, and auricular point sticking. The application of acupuncture combined with moxibustion was the most common intervention measure (Table 2). The use of moxibustion alone had the highest 
TABLE 2: Statistical analysis of classification of intervention measures in experimental groups.

\begin{tabular}{|c|c|c|}
\hline Serial number & Treatment methods & Frequency \\
\hline \multirow[t]{4}{*}{1} & Acupuncture and moxibustion therapy combined with drug treatment & Total of 29 articles ( $46 \%)$ \\
\hline & Acupuncture and moxibustion + Western medicine (13 articles) & \\
\hline & $\begin{array}{l}\text { Acupuncture and moxibustion }+ \text { traditional Chinese medicine (oral/enema) } \\
\text { (12 articles) }\end{array}$ & \\
\hline & $\begin{array}{l}\text { Acupuncture and moxibustion }+ \text { traditional Chinese medicine }+ \text { Western } \\
\text { medicine ( } 4 \text { articles) }\end{array}$ & \\
\hline \multirow[t]{9}{*}{2} & Single or combination use of acupuncture and moxibustion therapy & Total of 34 articles (54\%) \\
\hline & Acupuncture + moxibustion (17 articles) & \\
\hline & Moxibustion ( 9 articles) & \\
\hline & Balance cupping ( 1 article) & \\
\hline & Acupoint application ( 2 articles) & \\
\hline & Acupoint catgut embedding ( 2 articles) & \\
\hline & Acupuncture + massage (tui na) $(1$ article $)$ & \\
\hline & Acupuncture + TDP $(1$ article $)$ & \\
\hline & Abdominal acupuncture + catgut embedding ( 1 article $)$ & \\
\hline
\end{tabular}

TABLE 3: Statistical analysis of the use of moxibustion.

\begin{tabular}{lcc}
\hline $\begin{array}{l}\text { Serial } \\
\text { number }\end{array}$ & Moxibustion methods & $\begin{array}{c}\text { Number of } \\
\text { articles }\end{array}$ \\
\hline 1 & $\begin{array}{c}\text { Moxa stick } \\
\text { moxibustion } \\
\text { Ginger moxibustion } \\
\text { Moxibustion with } \\
\text { medicinal cakes } \\
\text { Warm acupuncture } \\
\text { Thunder fire } \\
4\end{array}$ & 18 \\
5 & $\begin{array}{c}\text { moxibustion } \\
\text { Direct moxibustion } \\
\text { Long snake } \\
\text { moxibustion } \\
7\end{array}$ & 7 \\
8 & $\begin{array}{c}\text { Heat-sensitive } \\
\text { moxibustion } \\
\text { Salt-separated } \\
\text { moxibustion }\end{array}$ & 1 \\
\hline
\end{tabular}

frequency, of which partitioned moxibustion was the most commonly used (Table 3).

3.3. Selection of Meridians and Acupoints. A statistical analysis of the frequency of acupoints used in acupuncture and moxibustion therapy in the experimental groups in the 63 included articles revealed the use of 55 acupoints in total. More than 10 articles used 12 acupoints, and the Tianshu (ST25) acupoint had the highest frequency of use (Table 4).

A statistical analysis of the meridian tropism, division, and specific point properties of all 55 acupoints showed that the clinical selection of acupoints for the treatment of UC using acupuncture and moxibustion involved a set of 10 regular meridians and 2 sets of an additional 8 extra-meridians.
TABLE 4: Statistical results of commonly used acupoints.

\begin{tabular}{lc}
\hline Acupoint & Frequency \\
\hline Tianshu (ST25) & 53 \\
Zusanli (ST36) & 49 \\
Guanyuan (RN4) & 42 \\
Shangjuxu (ST37) & 33 \\
Zhongwan (RN12) & 32 \\
Dachangshu (BL25) & 26 \\
Pishu (BL20) & 25 \\
Qihai (RN6) & 24 \\
Shenshu (BL23) & 19 \\
Shenque (RN8) & 17 \\
Yinlingquan (SP9) & 15 \\
Sanyinjiao (SP6) & 13 \\
\hline
\end{tabular}

TABLE 5: Statistical results of commonly used meridians.

\begin{tabular}{lc}
\hline Meridians & Number of points \\
\hline Yangming Stomach Channel of Foot & 13 \\
Taiyang Bladder Channel of Foot & 11 \\
Taiyin Spleen Channel of Foot & 10 \\
Ren Meridian & 8 \\
Kidney Channel of Foot-Shaoyin & 5 \\
Governor Meridian & 4 \\
Yangming Large Intestine Channel of Hand & 4
\end{tabular}

The most commonly used meridians were Yangming Stomach Channel of Foot, Taiyin Spleen Channel of Foot, Taiyang Bladder Channel of Foot, and Ren Meridian (Table 5).

The divisions mainly included the abdomen, back, and lower limbs. The utilization of specific points was as high as 
TABLE 6: Statistical results of total number of treatment days.

\begin{tabular}{lc}
\hline $\begin{array}{l}\text { Total number of } \\
\text { treatment days }\end{array}$ & $\begin{array}{c}\text { Number of } \\
\text { articles }\end{array}$ \\
\hline$<10$ days & 1 \\
10 19 days & 11 \\
20 29 days & 7 \\
$30 \sim 39$ days & 14 \\
$40 \sim 49$ days & 7 \\
$50 \sim 59$ days & 1 \\
$60 \sim 69$ days & 6 \\
70 days and above & 1 \\
Unknown & 10 \\
\hline
\end{tabular}

$69 \%$. The major points were five-shu point, back-shu point, lower He-Sea point, mu point, and convergent point. The composition of acupoints exhibited the following features: (1) distal-proximal point combinations involving the combined use of abdominal acupoints (proximal) and lower extremity acupoints (distal); (2) back-shu point and front-mu point combinations, such as the combined use of Tianshu (ST25) and Dachangshu (BL25), Zhongwan (RN12) and Weishu, and Guanyuan (RN4) and Xiaochangshu; and (3) acupuncture through meridians. In addition to the commonly used acupoints, the corresponding meridian points were used in combination mainly based on symptoms or the differentiation of symptoms.

Among the 63 included articles, 52 articles described the treatment frequency, the number of treatments in each treatment course, and the total number of treatment courses. There were 46 articles describing the use of acupuncture and moxibustion intervention measures in experimental groups once daily. The most common treatment course comprised 10 treatments. The number of total treatments varied from 10 days to more than 70 days. A statistical analysis using 10 days as one segment showed that the total treatment time was most often 30 days or 10-19 days. The duration of each acupuncture and moxa stick moxibustion treatment ranged from 15 to $40 \mathrm{~min}$; a treatment time of $30 \mathrm{~min}$ was the most common. Indirect moxibustion was generally applied for 3 Zhuangs. Some articles also reported 4-7 Zhuangs of moxibustion (Tables 6 and 7).

3.4. Efficacy Criteria and Outcome Indicators. The efficacy criteria and outcome indicators of the 63 included articles were statistically analyzed. Most articles used the efficacy criteria of the "1993 Taiyuan ulcerative colitis diagnostic criteria from the national conference on chronic noninfectious intestinal diseases," the "principles of guidelines for clinical studies of new drugs in traditional Chinese medicine," and self-formulated efficacy criteria (Table 8).

The outcome indicators included the total effectiveness rate, colon activity index, clinical symptom scores, fiber colonoscopy, laboratory indicators (including T lymphocyte subpopulations; immunoglobulins; tumor necrosis factor $\alpha$ (TNF $\alpha$ ); interleukin- (IL-) 6, 8, and 10; and C-reactive
TABLE 7: Statistical results of single treatment duration.

\begin{tabular}{lc}
\hline $\begin{array}{l}\text { Duration of a single } \\
\text { treatment (e.g., retaining } \\
\text { needles or moxibustion) }\end{array}$ & $\begin{array}{c}\text { Number of } \\
\text { articles }\end{array}$ \\
\hline$<20 \mathrm{~min}$ & 11 \\
$20 \sim 30 \mathrm{~min}$ & 30 \\
$>30 \mathrm{~min}$ & 6 \\
$\begin{array}{l}\text { Not calculated or } \\
\text { unknown }\end{array}$ & 16 \\
\hline $\begin{array}{l}{ }^{*} \text { Not calculated: moxa Zhuangs are used to indirectly describe the mox- } \\
\text { ibustion treatment time; unknown: a single treatment time is not clearly } \\
\text { described. }\end{array}$
\end{tabular}

protein), and adverse reactions to treatment measures. All 63 articles used the effectiveness rate as an outcome indicator. In addition, 10 articles [10, 29, 31, 40, 45, 47, 56-58, 61] used fiber colonoscopy as an outcome indicator, 16 articles used laboratory detection as an outcome indicator $[3,4,6,14,15$, $19,28,35,38-40,50,57-59,61], 3$ articles used colon activity as an outcome indicator [44, 56, 57], 8 articles used symptom scores as an outcome indicator $[10,19,21,38,44,55,57$, 61], and 3 articles used intestinal mucosal pathology as an outcome indicator $[8,13,36]$.

3.5. Clinical Trial Design. The included 63 articles contained a total of 5,404 cases of UC patients. There were 2,735 cases of male patients (50.6\%) and 2,152 cases of female patients (39.8\%). The gender of the other 517 patients in 7 articles was not reported. The average size of the trials was 87.8 cases; the smallest was 29 cases, and the largest was 640 cases.

An evaluation of the risk of bias showed that, among the 63 included RCT articles, 17 articles reported methods for the generation of random sequences [8-10, 16, 23, 27, $38,39,44,46-49,55,56,61,63]$, and 3 articles used proper allocation concealment $[8,39,44]$. The methods of random allocation and allocation concealment of the other trials were not appropriate or were unclear. Four articles reported using blind methods [28, 35, 39, 44]; however, the descriptions of these methods in two articles [28, 35] were unclear. Two studies $[39,44]$ reported the numbers of cases removed from the trials and the reasons for removal. Two trials performed an estimation of sample size before the study [39, 44]. The quality of the methodology and reporting of the included articles was generally low. Six studies reported follow-up conditions $[8,20,39,51,57,61]$. An evaluation of the quality of the 63 included articles using modified Jadad scoring showed that 3 articles $[8,39,44]$ represented high-quality literature.

\section{Discussion}

4.1. Diagnostic Standards of Clinical Studies on UC Treatment Using Acupuncture and Moxibustion. The correct diagnostic standards are a prerequisite for the implementation of clinical studies. With continuous improvements in the in-depth understanding of UC in medicine, the diagnostic standards for this disease are also being constantly refined. From 
TABLE 8: Utilization of efficacy assessment criteria.

\begin{tabular}{|c|c|c|}
\hline $\begin{array}{l}\text { Serial } \\
\text { number }\end{array}$ & Efficacy assessment methods & $\begin{array}{c}\text { Number of } \\
\text { articles }\end{array}$ \\
\hline 1 & $\begin{array}{l}1978 \text { Hangzhou conference on ulcerative diagnostic standards of the Chinese Society of Gastroenterology, } \\
\text { Chinese Medical Association }\end{array}$ & 1 \\
\hline 2 & $\begin{array}{l}1992 \text { standards for the diagnosis, symptom differentiation, and efficacy of chronic and nonspecific ulcerative } \\
\text { colitis (CUC) (provisional programs) of the Professional Committee on digestive diseases of the Shanxi Linfen } \\
\text { Symposium, Chinese Association of Integrative Medicine }\end{array}$ & 1 \\
\hline 3 & $\begin{array}{l}1993 \text { Taiyuan ulcerative colitis diagnostic criteria of the national conference on chronic noninfectious intestinal } \\
\text { diseases }\end{array}$ & 9 \\
\hline 4 & $\begin{array}{l}2000 \text { Chengdu conference on diagnostic standards for inflammatory bowel disease of the Chinese Society of } \\
\text { Gastroenterology, Chinese Medical Association }\end{array}$ & 3 \\
\hline 5 & $\begin{array}{l}2007 \text { consensus on standardization of diagnosis and treatment of inflammatory bowel disease in China by the } \\
\text { inflammatory bowel disease collaborative group of the Chinese Society of Gastroenterology, Chinese Medical } \\
\text { Association }\end{array}$ & 3 \\
\hline 6 & $\begin{array}{l}2009 \text { consensus on diagnosis and treatment of ulcerative colitis using traditional Chinese medicine of the } \\
\text { Digestive Diseases Branch of the Chinese Medical Association }\end{array}$ & 2 \\
\hline 7 & $\begin{array}{l}2010 \text { consensus on diagnosis and treatment of ulcerative colitis using traditional Chinese medicine of the } \\
\text { Digestive Diseases Branch of China Association of Traditional Chinese Medicine }\end{array}$ & 1 \\
\hline 8 & 《Principles of guidelines for clinical studies of new drugs in traditional Chinese medicine 》 & 11 \\
\hline 9 & 《Standards of efficacy and diagnosis of disease symptoms in traditional Chinese medicine 》 & 3 \\
\hline 10 & 90th National Integrative Medicine Symposium & 1 \\
\hline 11 & $\begin{array}{l}\text { Guidelines for diagnosis and treatment in integrative medicine (draft), Professional Committee on digestive } \\
\text { diseases, Chinese Association of Integrative Medicine }\end{array}$ & 1 \\
\hline 12 & English & 1 \\
\hline 13 & Teaching materials, books, and other literature & 7 \\
\hline 14 & None & 2 \\
\hline 15 & Self-formulated & 17 \\
\hline
\end{tabular}

Note: the 7 articles with "teaching materials, books, and other literature" as efficacy assessment methods included 1 article on "chronic colitis," 2 articles on "diagnosis of gastrointestinal diseases," 1 article on "principles of guidelines for new drugs," 1 article on "internal medicine," and 2 articles on "sulfasalazine rectal suppository collaborative group: comparative study of the treatment of ulcerative colitis using sulfasalazine using rectal suppositories and oral tabletsanalysis of 62 clinical cases."

the diagnosis of clinical manifestations to the combination of lesion features under colonoscopy, the application of pathological examinations, endoscopic mucosal staining techniques, and magnification endoscopy are increasing the scientific rigor of diagnosis. In addition, disease condition assessment is an important component of UC diagnosis; this includes clinical type, lesion range, disease activity, severity, parenteral manifestation, and complications. These factors all have an important influence on the formulation of treatment regimens and the determination of clinical efficacy. However, this study revealed that the current diagnostic standards used for the treatment of UC with acupuncture and moxibustion exhibit a high degree of randomness. Most of the clinical trials adopted relatively old diagnostic standards, and even more studies used diagnostic standards described in different types of teaching materials and books. The variety of standards used had an influence on the accuracy of patient inclusion and thus affected the reliability of the studies' conclusions. In addition, the majority of articles did not report the disease assessment conditions of the included cases, which reduced the repeatability of the studies' conclusions and their reference value for clinical decision-making.
Currently, most consensus opinions and diagnostic standards rely on Western medicine methods because the features of the lesions of UC patients in China are similar to those in Western countries. The UC lesion range classification in the newest (2012) edition of consensus also recommends the 2008 European Crohn's and Colitis Organisation (ECCO) consensus and the 2010 Asian Pacific Association of Gastroenterology (APAGE) consensus [64]. However, physicians who practice acupuncture and moxibustion therapies guided by traditional Chinese medicine theories also should pay attention to syndrome differentiation-dependent treatment during disease differentiation-dependent treatment. Therefore, the diagnostic standards of symptom differentiation classification in traditional Chinese medicine should be considered. In the past 3 years, 9 articles $[12,38,40,44,48,49$, $55,58,61]$ used the diagnostic standards of both traditional Chinese medicine and Western medicine, indicating that the diagnostic standards of traditional Chinese medicine have gradually received increasing attention. However, there are still fewer articles using the diagnostic standards of traditional Chinese medicine compared with Western medicine; the former needs attention from more researchers. 
4.2. Acupuncture and Moxibustion Therapy Programs for UC. This study showed that the intervention measures applied to experimental groups in clinical trials of treatment for UC using acupuncture and moxibustion involved either acupuncture and moxibustion therapy combined with drugs or the use of acupuncture and moxibustion therapy alone or in combination. The results showed that the treatment effects in experimental groups were better than those in control groups. The selection of acupoints in trials mostly involved acupoints belonging to Yangming Stomach Channel of Foot, Taiyin Spleen Channel of Foot, Taiyang Bladder Channel of Foot, and Ren Meridian. Specific acupoints in the abdomen, back, and lower extremities of the above meridians were mainly used. These features were consistent with records in ancient literature [65, 66]. Currently, treatment regimens for UC using acupuncture and moxibustion have the following drawbacks. (1) Insufficient attention is paid to the combination of symptom differentiation acupoints. Only 2 articles $[51,54]$ formulated corresponding acupoint selection programs based on different symptom types. During the implementation of a RCT, the intervention measures used in the same group should remain consistent; in addition, the individuality of acupuncture and moxibustion treatments should be considered; therefore, to ensure the consistency of major treatment methods, treatments should be properly adjusted based on different symptom types of patients. (2) The setting of treatment courses is based on personal experiences, which leads to randomness. The results of this study showed that the examined clinical studies of the treatment of UC using acupuncture and moxibustion had large differences in needle retention time, the number of treatments in a single treatment course, the total number of treatment courses, and the number of Zhuangs in moxibustion treatment. The treatment course is associated with the properties of the disease and the intervention measures. In the future, studies examining the best treatment course for UC using acupuncture and moxibustion should be implemented to optimize the duration and interval time of treatment courses and to increase clinical efficacy. (3) The targets of the intervention measures of acupuncture and moxibustion are lacking. In UC, the lesion locations differ between the rectum and colon, the disease severities differ, and the attack period and remission period differ; therefore, the intervention measures of acupuncture and moxibustion should also differ accordingly. However, none of the 63 included articles described lesion locations, stages, or disease severity. Therefore, it was unclear whether the acupuncture and moxibustion intervention measures adopted in these studies are applicable to patients with different conditions.

4.3. Efficacy Criteria and Outcome Indicators of Clinical Studies on the Treatment of UC Using Acupuncture and Moxibustion. Since 1978, the Chinese Society of Gastroenterology of the Chinese Medical Association has issued 5 versions of UC diagnosis and treatment standards and efficacy assessment indicator systems. The existing efficacy assessment indicators have developed gradually in the industry and provide very good guidance for clinical applications [67]. In addition to self-formulation, most of the articles included in this study used the 2002 "principles of guidelines for clinical studies of new drugs in traditional Chinese medicine (provisional)" [68]. The UC efficacy assessment indicators proposed by this edition of principles include comprehensive efficacy, mucosal lesion efficacy, syndrome efficacy, major symptom efficacy, and related laboratory detection indicators; these indicators cover both traditional Chinese medicine and Western medicine and are comprehensive. However, the results of this study showed that the application of efficacy criteria in recent clinical studies of UC treatment using acupuncture and moxibustion was not sufficiently unified or standardized; most studies used old or self-formulated criteria. The inflammatory bowel disease study group of the Chinese Society of Gastroenterology of the Chinese Medical Association formulated the newest "consensus on the diagnosis and treatment of inflammatory bowel disease" in 2012 [69] to update and modify efficacy assessment indicators. These guidelines combined clinical symptoms and endoscopic examination as efficacy determination indicators, including remission conditions, clinical efficacy determination (remission, effectiveness, and ineffectiveness), and recurrence conditions. However, these criteria were not adopted in the included articles. In addition, for the selection of clinical observation indicators, most studies adopted clinical efficacy to compare effects; therefore, the subjectivity of effect assessment was stronger. Objective indicators, such as clinical symptom scoring or endoscopic and pathological scoring, were relatively rare; therefore, efficacy determination in these studies had a significant subjective tendency, and the reliability of the efficacy of acupuncture and moxibustion was reduced. In terms of long-term efficacy, most studies did not perform long-term follow-up. The studies that did perform follow-up did not describe the conditions of losses to followup (the number of patients lost to follow-up and the reasons for loss to follow-up) or the follow-up methods; therefore, the long-term efficacy of the acupuncture and moxibustion therapies used in these studies could not be determined.

\section{Conclusion}

In summary, the experimental design, methods, diagnosis, treatment, and efficacy assessment should be improved to provide high-quality evidence for clinical decision-making. With the methods and results of medical researches, the treatment effect of acupuncture and moxibustion on UC will be better observed in the future.

\section{Competing Interests}

The authors declare that they have no competing interests.

\section{Authors' Contributions}

Jun Ji, Yan Huang, and Xia-Fei Wang contributed equally to the paper and they have the same rights. Jun Ji, Yan Huang, and Lu-Yi Wu made the concept of the review; Xia-Fei Wang, Zhe Ma, Huan-Gan Wu, Hui-Rong Liu, and Hyoyoung Im performed literature screening; Yan Huang and 
Zhe Ma established the database; Jun Ji and Jing Li wrote the manuscript together; Jun Ji, Lu-Yi Wu, and Jing Li approved the final version of the manuscript.

\section{Acknowledgments}

This work was supported by National Natural Sciences Foundation of China, nos. 81303033; National Basic Research Program of China (973 Program), nos. 2009CB522900 and 2015CB554501; Shanghai Municipal Commission of Health and Family Planning, no. 20134013; Shanghai Health System outstanding academic leader, no. XBR2013106; China Postdoctoral Science Foundation, no. 2015M570380.

\section{References}

[1] S. Ma and G. L. Zhang, "The observation of acupuncture combined with moxibustion on 60 cases with ulcerative colitis," Chinese Acupuncture \& Moxibustion, vol. 5, pp. 275-276, 1997.

[2] Y. Dao, "Clinical observation of drug combined with moxibustion on 46 cases of with ulcerative colitis," Journal of Sichuan Continuing Education College of MS, vol. 3, pp. 25-26, 1997.

[3] H. G. Wu, "Effect of moxibstion on ulcerative colitis and expression of HLA-DR antigen on epithelial cell of the colon," Acupuncture Research, vol. 24, no. 1, pp. 12-16, 1999.

[4] H. Q. Li, Y. Zhou, D. Y. Zhang et al., "Effect of moxibustion in a box at Shenque (CV8) shangxia ju xu (ST37, ST39) about chronic ulcerative colitis," Journal of Clinical Acupuncture and Moxibustion, vol. 24, no. 9, pp. 33-35, 2008.

[5] Y. X. Mo, X. F. Wang, W. R. Qu et al., "Observation on curative effect of Moxa casing moxibustion plus Chinese herbal medicine enema to treat patients with chronic ulcerative colitis," Chinese Nursing Research, vol. 24, no. 3, pp. 216-217, 2010.

[6] X. Guo and F. Guo, "Suspended moxibustion on heat-sensitive acupoints in the treatment of ulcerative colitis," China's Naturopathy, vol. 18, no. 2, p. 12, 2010.

[7] H. J. Yang, Q. L. Zhao, and S. G. Zhao, "Clinical observation on moxibustion at CV 8 point 50 cases of chronic colitis," Journal of Qilu Nursing, vol. 17, no. 34, p. 125, 2011.

[8] G. Y. Zhou and J. H. Jin, "Clinical observation on therapeutic effect of electroacupuncture plus moxibustion combined with medicine on ulcerative colitis," Chinese Archives of Traditional Chinese Medicine, vol. 26, no. 9, pp. 2069-2071, 2008.

[9] S. H. Han, Z. H. Xu, X. F. Chen, and Y. M. Mei, "Efficacy of buried seeds in the treatment of ulcerative colitis combined with auricular points," Journal of Nursing and Rehabilitation, vol. 11, no. 6, pp. 570-571, 2012.

[10] X. P. Jiang, "Efficacy of treating ulcerative colitis by abdominal acupuncture plus catgut embedding," Clinical Journal of Chinese Medicine, no. 17, pp. 37-39, 2012.

[11] J. H. Zhou, "The observation of ginger moxibustion on 34 cases with ulcerative colitis," Jiangsu Journal of Traditional Chinese Medicine, vol. 24, no. 10, pp. 44-45, 2003.

[12] H. Ding, H. Wang, T. Zhang et al., "Effects of ginger moxibustion on 30 patients with asdthenic splenonephro-yang of ulcerative colitis," Acta Academiae Medicinae CPAF, vol. 18, no. 6, pp. 509-511, 2009.

[13] H. G. Wu, L. B. Zhou, D. R. Shi et al., "Morphological study on colonic pathology ulcerative colitis treated by moxibustion," World Journal of Gastroenterology, vol. 6, no. 6, pp. 861-865, 2000 .
[14] L. J. Wen, "Clinical observation of indirect moxibustion treatment of chronic ulcerative colitis," Journal of Jiangxi College of Traditional Chinese Medicine, vol. 15, no. 2, pp. 35-36, 2003.

[15] S. M. Wang, L. Q. Zhang, and J. Sun, "Clinical efficacy on acupuncture treatment of ulcerative colitis," Qianwei Journal of Medicine, vol. 20, no. 2, p. 135, 2003.

[16] Y.-L. Xu, Y.-H. Du, X.-M. Xu, and T.-Y. He, "Observation on therapeutic effect of herb-partitioned spread moxibustion for treatment of chronic nonspecific ulcerative colitis," Chinese Acupuncture \& Moxibustion, vol. 30, no. 4, pp. 289-291, 2010.

[17] H. P. Zhang, "The observation of indirect moxibustion on 60 cases with chronic ulcerative colitis," Nei Mongol Journal of Traditional Chinese Medicine, vol. 31, no. 7, p. 79, 2012.

[18] L. L. Chi and M. M. Yu, "Jian Pi Yu Yang decoction and Shenque sticking with sulfasalazine treatment of 44 cases of chronic ulcerative colitis," Journal of Practical Traditional Chinese Internal Medicine, vol. 25, no. 8, pp. 55-57, 2011.

[19] Y. H. Luo, "Balance cupping therapy in the treatment of ulcerative colitis for 40 cases," Jiangxi Journal of Traditional Chinese Medicine, vol. 40, no. 2, pp. 63-64, 2009.

[20] D. P. Li, Q. Chen, L. Yi et al., "Warm musk umbilical paste sticking with the electromagnetic wave radiation treatment of 40 cases of chronic ulcerative colitis," Shaanxi Journal of Traditional Chinese Medicine, vol. 27, no. 1, pp. 49-50, 2006.

[21] J. R. Tian, X. J. Li, H. W. Han et al., "Shenque acupoint sticking therapy in the treatment of chronic nonspecific ulcerative colitis clinical observation," Hebei Journal of Traditional Chinese Medicine, vol. 34, no. 6, pp. 884-885, 2012.

[22] J. Chen, "Clinical observation of chronic nonspecific ulcerative colitis by specific acupoints catgut embedding," Sichuan Journal of Traditional Chinese Medicine, vol. 22, no. 5, pp. 89-90, 2004.

[23] H.-J. Li, G.-P. Li, and H.-Y. Li, "Clinical observation on acupoint catgut embedding therapy for treatment of ulcerative colitis," Chinese Acupuncture \& Moxibustion, vol. 26, no. 4, pp. 261-263, 2006.

[24] D. J. Duan, L. N. Zhang, J. T. Yin et al., "Clinical observation on acupoint catgut embedding therapy combined with modified Bai Tou Weng decoction enema for treatment of chronic nonspecific ulcerative colitis," Hebei Journal of Traditional Chinese Medicine, vol. 34, no. 5, pp. 721-722, 2012.

[25] Y. T. Sun and S. L. Wang, "Warming needle moxibustion combined with retention enema in the treatment of ulcerative colitis for 45 cases," Chinese Acupuncture Moxibustion, no. 9, p. 50, 1998.

[26] S. L. Wang, "Observation on therapeutic effect of warming needle therapy on ulcerative colitis," Chinese Community Doctors, no. 22, pp. 132-133, 2008.

[27] T. A. Ma and Z. K. Xu, "Observation on therapeutic effect of acupuncture with TDP on chronic ulcerative colitis," Central Plains Medical Journal, vol. 32, no. 17, pp. 31-32, 2005.

[28] J. Cui, "Study the effect of acupuncture on cytokines of ulcerative colitis patient," Hebei Medical Journal, vol. 32, no. 11, pp. 1409-1410, 2010.

[29] X. Y. Guo, Z. J. Long, X. Wu, and J. Cao, "Efficacy observation on treating 22 cases of UC by acupuncture combined with herbal retention enema," Liaoning Journal of Traditional Chinese Medicine, vol. 34, no. 5, pp. 654-655, 2007.

[30] D. M. Wang, X. G. Zhang, K. Chen et al., "Acupuncture combined with retention enema with combination of Chinese and western drugs in the treatment of ulcerative colitis," Practical Pharmacy and Clinical Remedies, vol. 12, no. 3, pp. 224-225, 2009. 
[31] Z. J. Long and X. L. Yang, "Acupuncture combined with retention enema in the treatment of ulcerative colitis for 23 cases," Chinese Journal of Coloproctology, vol. 30, no. 2, pp. 5859, 2010.

[32] H. Y. Chen, "Clinical observation and nursing on acupuncture combined with TCM retention enema for ulcerative colitis," Chinese and Foreign Medical Research, vol. 8, no. 12, pp. 101-102, 2010.

[33] G. H. Sun and X. L. Wang, "Acupuncture for chronic ulcerative colitis in 35 cases," Journal of Practical Traditional Chinese Medicine, vol. 17, no. 6, p. 32, 2001.

[34] S. M. Wang, X. G. Li, L. Q. Zhang et al., "Clinical study on drug-separated moxibustion at Shenque (CV8) for treatment of ulcerative colitis," Chinese Acupuncture \& Moxibustion, vol. 26, no. 2, pp. 97-99, 2006.

[35] Y. Q. Shi, S. P. Liu, and J. G. Liu, "The influence of acupuncture and moxibustion therapy on cell factors of ulcerative colitis patients," Hubei Journal of Traditional Chinese Medicine, vol. 28, no. 2, pp. 11-12, 2006.

[36] Q. Shen, J. Lu, and S. M. Guo, "Observations on the efficacy of acupuncture at crossed points as main treatment for ulcerative colitis," Shanghai J Acu-Mox, vol. 31, no. 5, pp. 319-320, 2012.

[37] Q. J. Xu, "56 cases of chronic ulcerative colitis treated with the integrated Chinese and western medicine therapy," Journal of Chinese Physician, vol. 34, no. 1, pp. 48-49, 2006.

[38] Z. J. Zhang, S. Xie, G. F. Ma et al., "Effect of TCM on the expression of IL-8, IL-10 in patients with ulcerative colitis," Shanxi Journal of Traditional Chinese Medicine, vol. 32, no. 5, pp. 566-568, 2011.

[39] S. Joos, N. Wildau, R. Kohnen et al., "Acupuncture and moxibustion in the treatment of ulcerative colitis: a randomized controlled study," Scandinavian Journal of Gastroenterology, vol. 41, no. 9, pp. 1056-1063, 2006.

[40] C. Yang and H. Yan, "Observation of the efficacy of acupuncture and moxibustion in 62 cases of chronic colitis," Journal of Traditional Chinese Medicine, vol. 19, no. 2, pp. 111-114, 1999.

[41] S. Ma, "Observation on the therapeutic effect of combined treatment of 60 cases of ulcerative colitis with acupuncture and moxibustion," World Journal of Acupuncture-Moxibustion, vol. 9, no. 1, pp. 24-26, 1999.

[42] X. Ma, "Acupuncture treatment for 76 cases of ulcerative colitis," Journal of Traditional Chinese Medicine, vol. 25, no. 4, pp. 264265, 2005.

[43] Z. Wang, "46 cases of ulcerative colitis treated by warming needle moxibustion," China Foreign Medical Treatment, vol. 32, no. 34, pp. 126-128, 2013.

[44] Z. Zhong, A Clinical Randomized Controlled Trial on Acupuncture with Strenthening Spleen and Kidney Method for Ulcerative Colitis, Chengdu University of Traditional Chinese Medicine, 2013.

[45] X. B. Hu, "Efficacy observation on treating 50 cases of UC by acupuncture combined with herbal retention enema," Clinical Journal of Chinese Medicine, vol. 16, pp. 49-50, 2013.

[46] W. Y. Li, "Clinical observation of Bupi Yichang pill and warming needle moxibustion in the treatment of chronic ulcerative colitis with spleen and kidney deficiency," Information on Traditional Chinese Medicine, vol. 31, no. 6, pp. 87-89, 2014.

[47] Y. Ou, E. N. Wu, and J. C. Xu, "Clinical observation on treating 35 cases of ulcerative colitis with spleen deficiency by warming needle moxibustion," Chinese Journal of Ethnomedicine and Ethnopharmacy, vol. 23, no. 24, p. 55, 2014.
[48] C. Y. Wang, Y. Feng, L. Feng et al., "Clinical effects of acupuncture with Chinese and western medicine treating on ulcerative colitis," Medical Journal of West China, vol. 26, no. 2, pp. 199203, 2014.

[49] X. W. Ma, L. J. Deng, Y. M. Cao et al., "Acupuncture combined massage Three-step Nine method for the treatment of chronic ulcerative colitis random parallel control study," Journal of Practical Traditional Chinese Internal Medicine, no. 4, pp. 151153, 2014.

[50] B. Sun, "Effect of acupuncture and moxibustion to T cell subset with ulcerative colitis," Asia-Pacific Traditional Medicine, vol. 11, no. 24, pp. 104-105, 2015.

[51] Z. Y. Wu, "Comparison of curative effect of chronic nonspecific ulcerative colitis treated by acupuncture combined with traditional Chinese medicine," Journal of Zhejiang Chinese Medical University, no. 4, pp. 315-316, 2015.

[52] Y. Mi, "Efficacy observation on treating chronic ulcerative colitis by acupuncture and moxibustion," Nei Mongol Journal of Traditional Chinese Medicine, vol. 34, no. 3, pp. 50-51, 2015.

[53] T. M. Mo, "Curative effect of chronic ulcerative colitis treated by acupuncture and moxibustion," Yunnan Journal of Traditional Chinese Medicine and Materia Medica, vol. 36, no. 11, pp. 96-97, 2015.

[54] X. Y. Huang, X. Q. Chen, and Q. Z. Zhang, "Efficacy observation on treating chronic ulcerative colitis by TCM acupuncture and moxibustion," Chinese Journal of Modern Drug Application, vol. 9, no. 13, pp. 255-256, 2015.

[55] W. Q. Dong, Clinical Observation of Herb-Separated Moxibustion in the Treatment of Ulcerative Colitis with Spleen-Kidney Yang Deficiency, Beijing University of Chinese Medical, 2014.

[56] Z. S. He and S. K. Zhong, "Treatment of mild to moderate unlcerative colitis with salazosulfapyridine and thunder-fire moxibustion in 30 cases," Journal of Changchun University of Traditional Chinese Medicine, vol. 29, no. 2, pp. 1051-1053, 2013.

[57] X. Wang, Clinical Observation of Warming and Tonifying the Spleen and Kidney Combined with Moxibustion in Treating Ulcerative Colitis of Spleen-Kidney Yang Deficiency, Nanjing University of Chinese Medicine, Nanjing, China, 2014.

[58] G. Y. Han, G. Y. Wang, J. Lu et al., "Mechanisms of combined treatment of warm needling and Wenchang decoction retention for ulcerative colitis," Journal of Clinical and Experimental Medicine, no. 23, pp. 1981-1984, 2015.

[59] J. He, M. X. Liao, and Z. Z. Meng, "Therapeutic effect of acupuncture combined with umbilical ring point gingerpartition moxibustion for ulcerative colitis of spleen-kidney yang deficiency type and its influence on related inflammatory factors," Journal of Guangzhou University of Traditional Chinese Medicine, no. 4, pp. 687-693, 2015.

[60] X. Quan, "96 Cases of chronic ulcerative colitis treated by acupuncture and double-mox," Hunan Journal of Traditional Chinese Medicine, vol. 31, no. 5, pp. 89-90, 2015.

[61] K. Chen and C. Li, "Acupuncture combined with medication for ulcerative colitis with damp-heat syndrome at active phase," Chinese Acupuncture \& Moxibustion, vol. 35, no. 5, pp. 435-438, 2015.

[62] F. Ge, Y. Ji, X. P. Ma et al., "Clinical effect observation of electroacupuncture combined with medicine in the treatment of ulcerative colitis," China Modern Medicine, no. 6, pp. 164-168, 2015.

[63] L. P. Wei, H. Q. Song, and D. B. Ye, "30 Cases of ulcerative colitis with spleen kidney yang deficiency," Chinese Medicine Modern Distance Education of China, vol. 12, no. 15, pp. 129-130, 2014. 
[64] M. Wang and X. S. Wang, "Diagnosis and treatment of ulcerative colitis changes," Chinese Journal of Clinical Gastroenterology, no. 5, pp. 315-317, 2015.

[65] X. M. Wang, H. G. Wu, H. R. Liu et al., "TCM etiology and pathogenesis of ulcerative colitis and acupuncture acupoints features review," Liaoning Journal of Traditional Chinese Medicine, vol. 34, no. 7, pp. 891-893, 2007.

[66] L. Q. Meng, L. X. Ji, and Y. T. Cheng, "Study on ancient literatures about acupuncturing on the treatment of similar symptoms of ulcerative colitis," Journal of Shanxi College of Traditional Chinese Medicine, no. 1, pp. 1-3, 2014.

[67] H. Shen, L. Zhu, and L. Zhang, "Strategy of evaluation of Chinese medical curative effect on ulcerative colitis," Journal of Traditional Chinese Medicine, vol. 57, no. 2, pp. 126-130, 2016.

[68] X. Y. Zheng, TCM New Drug Clinical Research Guidelines (Trial), Chinese Medical Science Press, Beijing, China, 2002.

[69] IBD Group with CSGE, "Diagnosis and treatment of inflammatory bowel disease consensus," Journal of Internal Medicine, vol. 51, no. 21, pp. 818-831, 2012. 


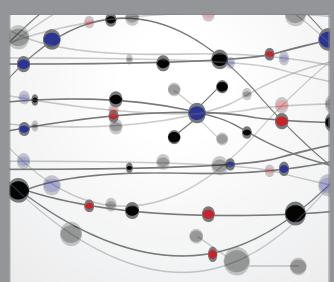

The Scientific World Journal
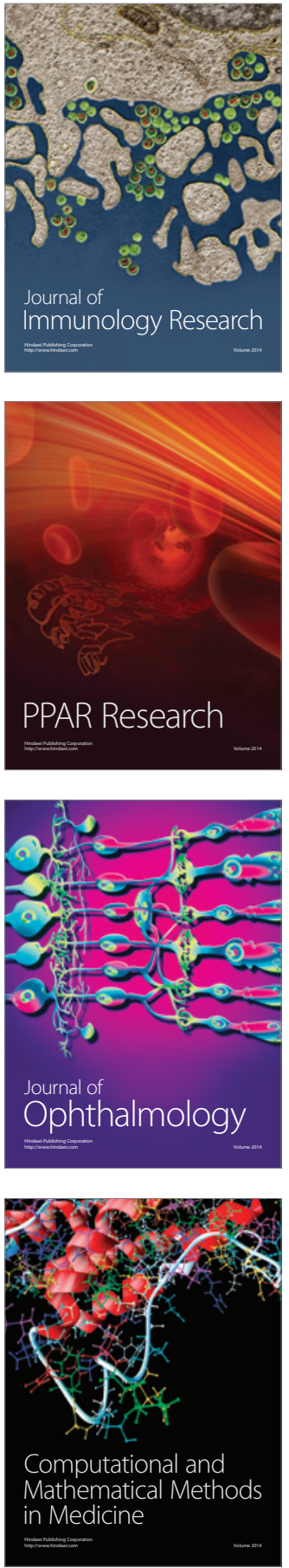

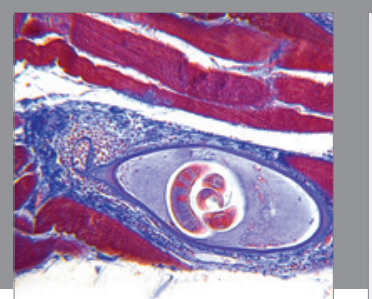

Gastroenterology Research and Practice

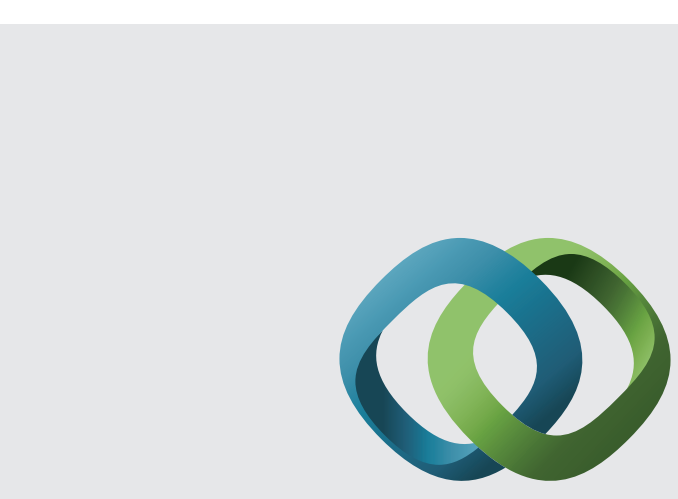

\section{Hindawi}

Submit your manuscripts at

http://www.hindawi.com
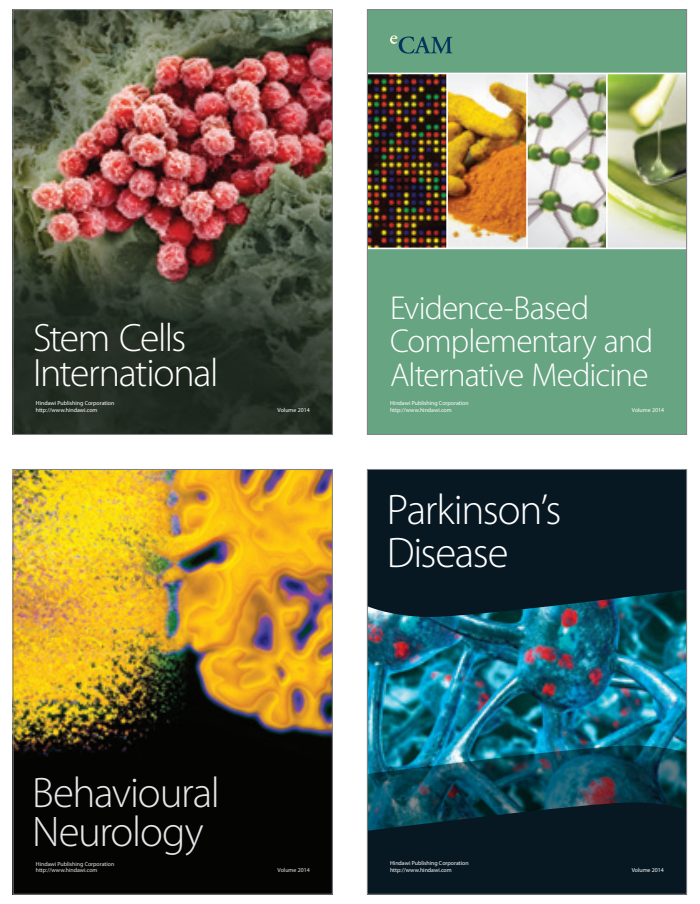
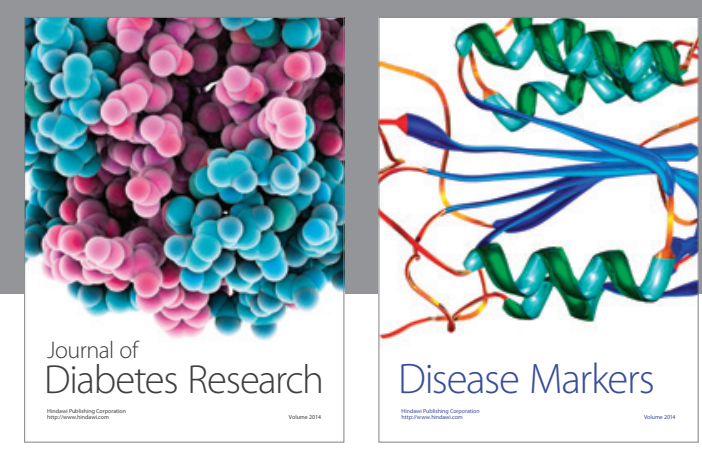

Disease Markers
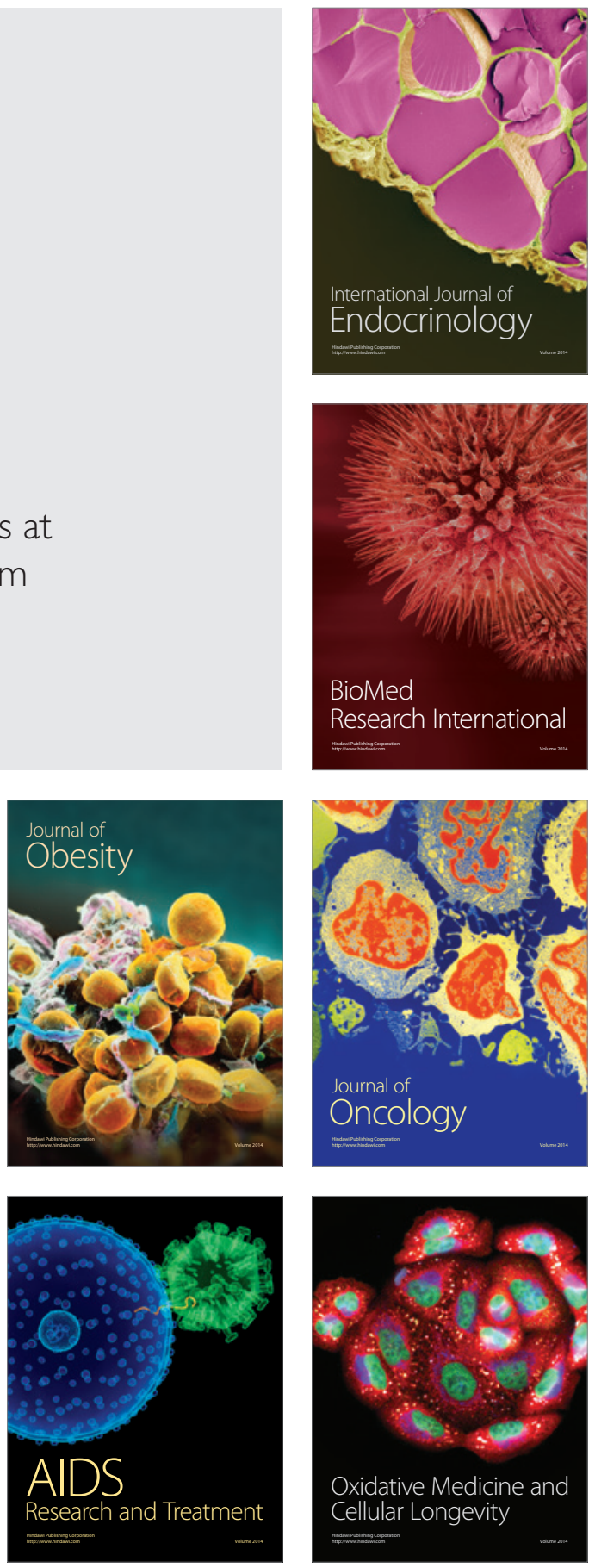